\title{
EXPERIMENTAL CHARACTERIZATION OF AN OVERDENSE PLASMA IN A COMPACT ION SOURCE
}

\author{
G. Castro $^{a, *}$, D. Mascali ${ }^{a}$, F. P. Romano ${ }^{a, b}$, C. Altana ${ }^{a, c}$, C. Caliri ${ }^{a, c}$, \\ L. Celona ${ }^{a}$, R. Di Giugno ${ }^{a, c}$, N. Gambino ${ }^{a, d}$, D. Lanaia ${ }^{a, e}$, \\ R. Miracoli ${ }^{a, f}$, A. Musumarra ${ }^{a, c}$, L. Neri $^{a}$, G. Torrisi ${ }^{a, g}$, S. Gammino ${ }^{a}$ \\ a INFN- Laboratori Nazionali del Sud, via S.Sofia 62, 95123, Catania, Italy \\ ${ }^{b}$ CNR-IBAM Via Biblioteca 4, 95124, Catania, ITALY \\ ${ }^{c}$ Università degli Studi di Catania, Dipartimento di Fisica e Astronomia, V. S.Sofia 64, 95123 Catania, Italy \\ ${ }^{d}$ ETH, Zurich, Switzerland \\ e CERN, Geneva, Switzerland \\ $f$ ESS Bilbao, Spain \\ $g$ Università Mediterranea di Reggio Calabria, DIIES, Via Graziella,I-89100, Reggio Calabria, Italy \\ * corresponding author: castrog@lns.infn.it
}

Abstract. Electron Cyclotron Resonance Ion Sources (ECRIS) are compact plasma-based machines able to feed particle accelerators with high intensity beams of multi-charged ions. ECRIS plasmas are density-limited, since they are sustained by E.M. wave propagation up to a cut-off density value. In the past, the only way to improve ECRIS performance was to increase the microwave frequency and the magnetic field strength to satisfy the ECR condition. A different plasma heating mechanism is being applied at INFN-LNS. It is based on Electron Bernstein Waves (EBW), i.e., electrostatic waves which do not suffer any density cut-off. Highlights concerning preliminary signatures of EBW formation and subsequent absorption are given here.

KEYWORDS: plasma; electrostatic waves; Bernstein waves; ion sources; ECRIS..

\section{INTRODUCTION}

Electron Cyclotron Resonance Ion Sources (ECRIS) are able to generate high-intensity highly-charged ion beams for accelerators, characterized by high reliability and low maintenance [1, 2]. An electromagnetic field in the microwave range is injected into a metallic chamber, where a magnetic field is generated. If somewhere in the chamber the microwave frequency $\omega_{c}$ is equal to the Electron Larmor frequency (i.e., $\omega_{c}=e B / m$, where $e$ is the electron charge and and $m$ is mass), then a large quantitative of energy is transferred from the wave to the free electrons present in the chamber. This mechanism is commonly called Electron Cyclotron Resonance (ECR), and it enables ECR plasmas to be generated and sustained. The ion content is finally removed by means of the system of high voltage electrodes generating the ion beam. Both the extracted current and the mean charge state are proportional to the electron density of the plasma generated in the source. To improve the performance of an ECRIS source, a continuous increase in the electron density is essential. Unfortunately, the maximum density of plasma that can be obtained in an ECRIS is limited. Microwaves (and in general any EM field) are reflected if the density exceeds a limit density value called the cut-off density ( $\left.n_{\text {cut-off }}\right)$. The cut-off density depends on the microwave frequency $f$, as $n_{\text {cut-off }}=\varepsilon_{0} m f 2 / e 2\left(\varepsilon_{0}\right.$ is the dielectric constant in a vacuum). In principle, then, the only way to increase the electron density is to increase the microwave fre- quency and consequently the magnetic field, in order to allow ECR. These considerations were first expressed by Geller, and are usually called "scale laws" [1]. Scaling laws have permitted the development of ECRIS in the last two decades, together with the so-called High-BMode principle [3], leading to a continuous increase in the strength of the magnetic field, which has taken the ancillary technologies near to their technological limits. It is reasonable to assume that the further development of ECRIS will become harder and harder, and the development of new plasma heating mechanisms that can overcome current constraints must be taken into consideration. ElectroStatic (ES) waves are compression and rarefaction waves that can be generated within plasma. Because of their own nature, they do not suffer any density cut-off, and can propagate for any electron density value [4. A particular kind of ES waves, generated only in magnetized plasmas and named Electron Bernstein Waves (EBW), in honour of Ira Bernstein, who first theorised their existence [5], is strongly absorbed by plasma at cyclotron harmonics, i.e., when $B=B_{\mathrm{ECR}} / n$ (where $n$ is a natural number). The dispersion relation of the Bernstein waves is in fact [6]:

$$
\begin{aligned}
\left(\frac{k_{B} v_{\mathrm{th}}}{\omega_{p}}\right)^{2}=1 & -\mathrm{e}^{-k_{B}^{2} r_{L}^{2}} \mathrm{I}_{q}\left(k_{b}^{2} r_{L}^{2}\right) \\
& -2\left(\frac{\omega}{\omega_{c}}\right)^{2} \frac{\sum_{q} \mathrm{e}^{-k_{B}^{2} r_{L}^{2}} \mathrm{I}_{q}\left(k_{B}^{2} r_{L}^{2}\right)}{n^{2}-\omega^{2} / \omega_{c}^{2}},
\end{aligned}
$$



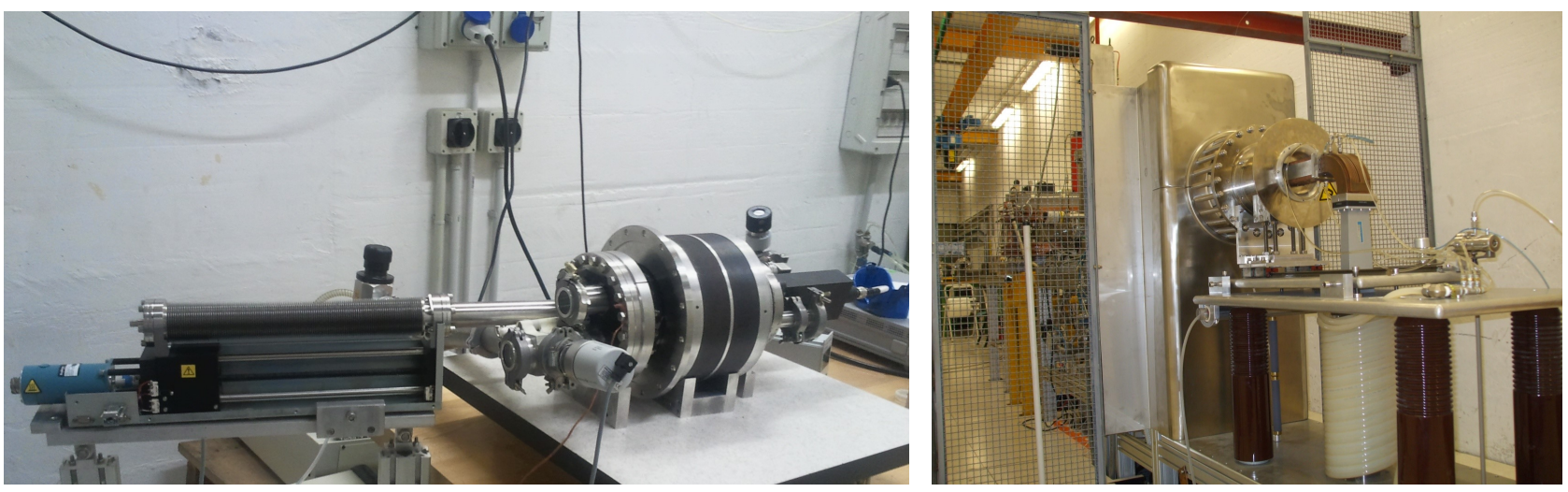

Figure 1. A view of the plasma reactor (left); a view of the VIS source (right).

where $k_{B}$ is the wave number of the Bernstein wave, $v_{\text {th }}$ is the electron thermal velocity, $r_{L}$ is the electron Larmor radius, $\omega_{p}$ and $\omega_{c}$ are the plasma and cyclotron frequencies, $\omega$ is the pumping frequency, and $\mathrm{I}_{q}$ is the Bessel function. The value of $k_{B}$ becomes infinite when the denominator of the previous equation is equal to zero, i.e., at cyclotron harmonics.

EBWs cannot be generated outside the plasma, and need to be internally triggered by EM waves. It can be shown that $\mathrm{X}$ waves convert into EBW and ion waves at Upper Hybrid Resonance (UHR) [7], when $\omega_{\mathrm{RF}}=$ $\sqrt{\omega_{p}^{2}+\omega_{c}^{2}}$. UHR can exist only if simultaneously $B<$ $B_{\mathrm{ECR}}$ and $n_{e}<n_{\text {cut-off }}$, so EBW can be generated in a plasma only if the magnetic field is below resonance $\left(B<B_{\mathrm{ECR}}\right)$ and the electron density is under-dense $\left(n_{e}<n_{\text {cut-off }}\right)$ somewhere in the plasma chamber.

The EM-to-ES conversion mechanism is expected to produce non-linear processes. They appear as additional components in the wave spectrum. The standing wave nature of the electromagnetic energy, which is additionally amplified in the proximity of UHR, could generate some "zero-frequency" oscillations able to excite ion sound waves at very low frequency [7].

The EBW resonance mechanism has been explained by the Segdeev and Shapiro model [8]; the absorption of EBW leads to the generation of hot electron layers [6], characterized by electron energies much higher than the usual ECR energies [9]. In conclusion, three different physical phenomena can be considered as fingerprints of EBW generation and absorption in plasma:

- Generation of supra-thermal electrons;

- Exceeding the cut-off density;

- Evidence of the existence of non-linear phenomena within the plasma;

In recent years, EBW heating has been successfully studied in large devices for fusion [10, 11]. Experimental evidence shows that is possible to generate EBW by means of the so-called OXB mechanism: by launching O-Waves with a particular angle with respect to the magnetic field, it is possible to trigger the generation of EBW from X-waves at UHR [10].
This new heating mechanism is being studied and developed for the first time at INFN-LNS in compact, plasma-based ion sources, where the modal behaviour of the E.M. waves can no longer be neglected (as in the fusion case). The tests were performed in an attempt to confirm EBW generation in ion sources for accelerators through a study of the three main fingerprints listed above. It is reasonable to assume that EBW heating could provide large benefits for the production of high-current light ions. Light ion generation does not in fact require strong constraints in the magnetic field profile, as highly-charged heavy ions perform the required high-B mode operations $[3]$.

\section{EXPERIMENTAL SET-UP}

The measurements presented in this paper were carried out in two different Microwave Discharge Ion Sources [12], featuring different magnetic profiles. The first source is a plasma reactor, consisting of a stainlesssteel cylinder $24 \mathrm{~cm}$ in length and $14 \mathrm{~cm}$ in diameter. An NdFeB permanent magnet system generates an offresonance magnetic field along the axis of the plasma chamber (with a maximum of $0.1 \mathrm{~T}$ on the axis). The second source is the Versatile Ion Source [13] (VIS), consisting of a water-cooled copper plasma chamber (100 $\mathrm{mm}$ in length and $90 \mathrm{~mm}$ in diameter). VIS enables us to have purely off-resonance microwave injection (which is not possible using a plasma reactor). Figure 1 shows a view of the two sources. Microwaves were generated using a conventional $2 \mathrm{~kW}$ magnetron, able to generate $2.45 \mathrm{GHz}$ microwaves, or a Travelling Wave Tube (TWT), able to generate microwaves from 3.2 to $7 \mathrm{GHz}$. The typical working frequency when using TWT was $3.7478 \mathrm{GHz}$. A movable Langmuir Probe (LP) was used to measure the plasma temperature and density. LP can host a small wire used as a local electromagnetic antenna, which can be connected to a spectrum analyser for spectral emission analysis of the plasma. An Si-Pin and an HPGe X-ray detector were used for measuring the X-ray spectra in various plasma conditions. Both detectors are able to detect $\mathrm{X}$-rays in the energy range from $1-30 \mathrm{keV}$. 


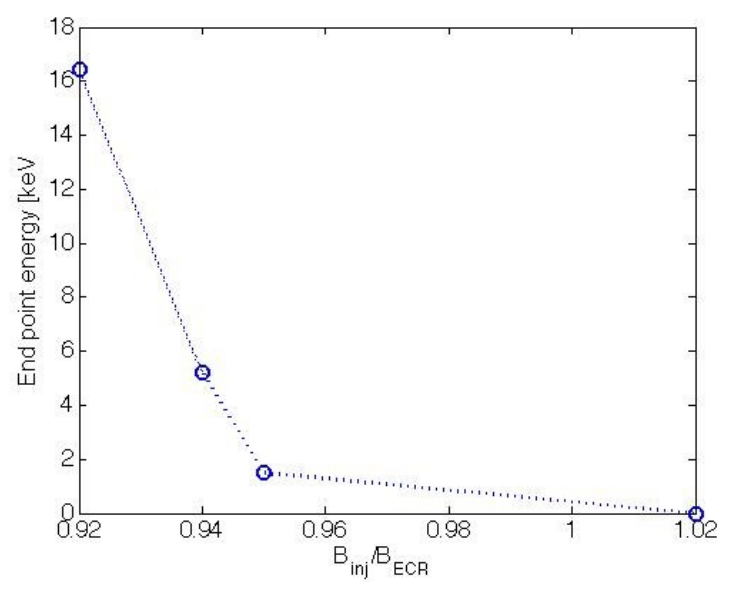

FiguRE 2. Spectral end-point energy with respect to the ratio of the magnetic field and microwave injection and ECR magnetic field $B_{\text {inj }}<B_{\mathrm{ECR}}$.

\section{Discussion}

Analyses of the Bremsstrahlung spectrum generated by electrons when colliding with the other plasma particles provide a tool for diagnosing high temperature electrons [14, 15]. In general, if the X-ray spectrum is known, it is possible to find the electron density and the temperature of the plasma that generated it [14. In addition, the spectrum end point represents roughly the highest electron energy reached in the plasma. In the case of ECR heating, the maximum energy $T_{\max }$ achievable by electrons can be calculated by means of Canobbio theory [16]:

$$
T_{\max }=1.5 \cdot 10^{9}(E / \omega)^{2 / 3}
$$

where $E$ is the maximum electric field in the plasma chamber, and $\omega$ is the microwave frequency. No confining magnetic field is present in MDIS, so the electrons cross the ECR surface only once or a few times. In such a case, by taking into account the typical working $\mathrm{RF}$ power in VIS and the plasma reactor $(<1000 \mathrm{~W})$ and the chamber volumes, it can be verified that the maximum electron energy $T_{\max }$ does not exceed 100 $200 \mathrm{eV}$. Indeed, electron energies higher than $200 \mathrm{eV}$ cannot be explained by means of ECR heating. Reference [17] shows the results of a series of measurements carried out on the VIS source at $2.45 \mathrm{GHz}$. The Xray spectrum, obtained with an Si-Pin detector, was studied for different strengths of the magnetic field at microwave injection. The magnetic field was modified by shifting the permanent magnets with respect to the plasma chamber, as shown in [17]. The plot of the end point energy against $B_{\mathrm{inj}} / B_{\mathrm{ECR}}$, shown in Figure 2 demonstrates that the presence of high energy X-rays is strictly related to the strength of the magnetic field when microwave injection $B_{\text {inj }}$ takes place. High-energy electrons are generated only if the magnetic field when the microwaves are injected is below resonance, i.e., $B_{\mathrm{inj}}<B_{\mathrm{ECR}}$, and the X-ray end point energy (and indeed the maximum electron

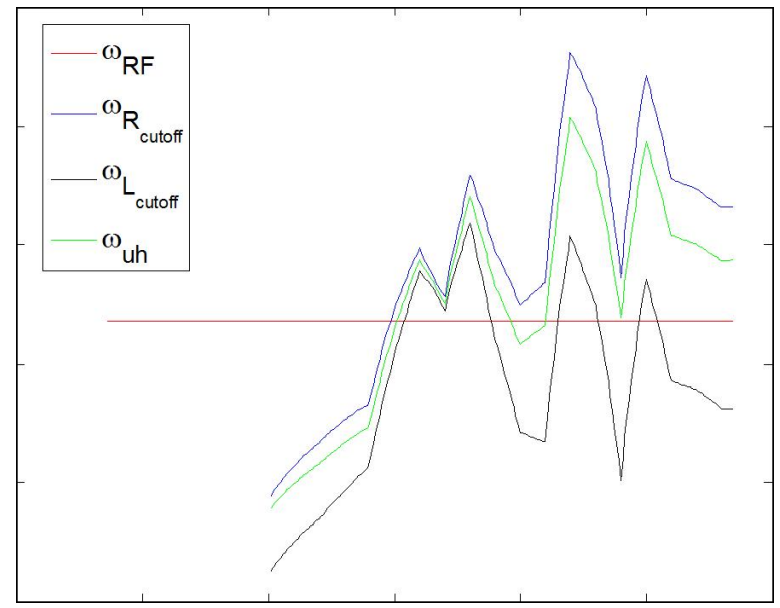

FigURE 3. Value of $\omega_{\mathrm{R}, \text { cut-off }}, \omega_{\mathrm{L}, \text { cut-off, }}$ and $\omega_{\mathrm{UH}}$ with respect to the position of the probe. $\omega_{\mathrm{RF}}=2 \pi$. $.3 .7576 \mathrm{GHz}$ crosses $\omega_{\mathrm{UH}}$ at 20 and $24 \mathrm{~cm}$ LP position, enabling UHR.

energy).increases progressively as $B_{\mathrm{inj}}$ drops lower than $B_{\mathrm{ECR}}$. The revealed end point energy values are not explainable by means of (2), so these electrons are not accelerated by ECR heating, but by a different heating mechanism. Furthermore, this heating mechanism is triggered by $B_{\mathrm{inj}}<B_{\mathrm{ECR}}$, which is a required condition for UHR to be placed somewhere inside the plasma, totally fitting what is expected for EBW heating.

A series of LP measurements within the chamber of the plasma reactor were carried out to obtain the electron density and temperature [17. The dimensions of the plasma chamber and the permanent magnet make it possible to have larger under-resonance and off-resonance volumes reachable by LP. The measurements were carried out at $1.5 \cdot 10^{-3}$ mbar and $2.45 \mathrm{GHz}$ pumping frequency, with the microwave power varying between 20 and $200 \mathrm{~W}$. Preliminary results are shown in [17]: the electron density is drastically enhanced in regions where the $B<B_{\mathrm{ECR}}$ condition is satisfied. This effect is observed for all the power values that we used; in particular, at $200 \mathrm{~W}$, electron density of about $1.5 \cdot 10^{12} \mathrm{~cm}^{-3}$ was obtained, a value twenty times greater than the cut-off density at $2.45 \mathrm{GHz}$ $\left(n_{\text {cut-off }}=7.5 \cdot 10^{10} \mathrm{~cm}^{-3}\right)$. In order to test pure EBW heating, $3.7576 \mathrm{GHz}$ microwaves were injected into the Plasma Reactor. The results of experimental measurements carried out with only $30 \mathrm{~W}$ injected power show a maximum electron density twice larger than ncutoff in the proximity of the cyclotron harmonics [18]. Simultaneous knowledge of the magnetic field and the electron density of the plasma reactor enables us to obtain the positioning of various plasma resonances and cut-offs. Figure 3 shows the values of $\omega_{\mathrm{R} \text {,cut-off, }}$ $\omega_{\mathrm{L}, \text { cut-off }}$, and $\omega_{\mathrm{UH}}$ with respect to the LP penetration length $\left(\omega_{R, \text { cut-off }}\right.$ is the frequency needed for the $R$ wave cut-off, $\omega_{\mathrm{L}, \text { cut-off }}$ is the frequency needed for the $\mathrm{L}$ wave cut-off, and $\omega_{\mathrm{UH}}$ is the frequency needed for UHR and $\left.\omega_{\mathrm{RF}}=2 \pi \cdot 3.7576 \mathrm{GHz}\right)$. $\omega_{\mathrm{RF}}$ cuts across 

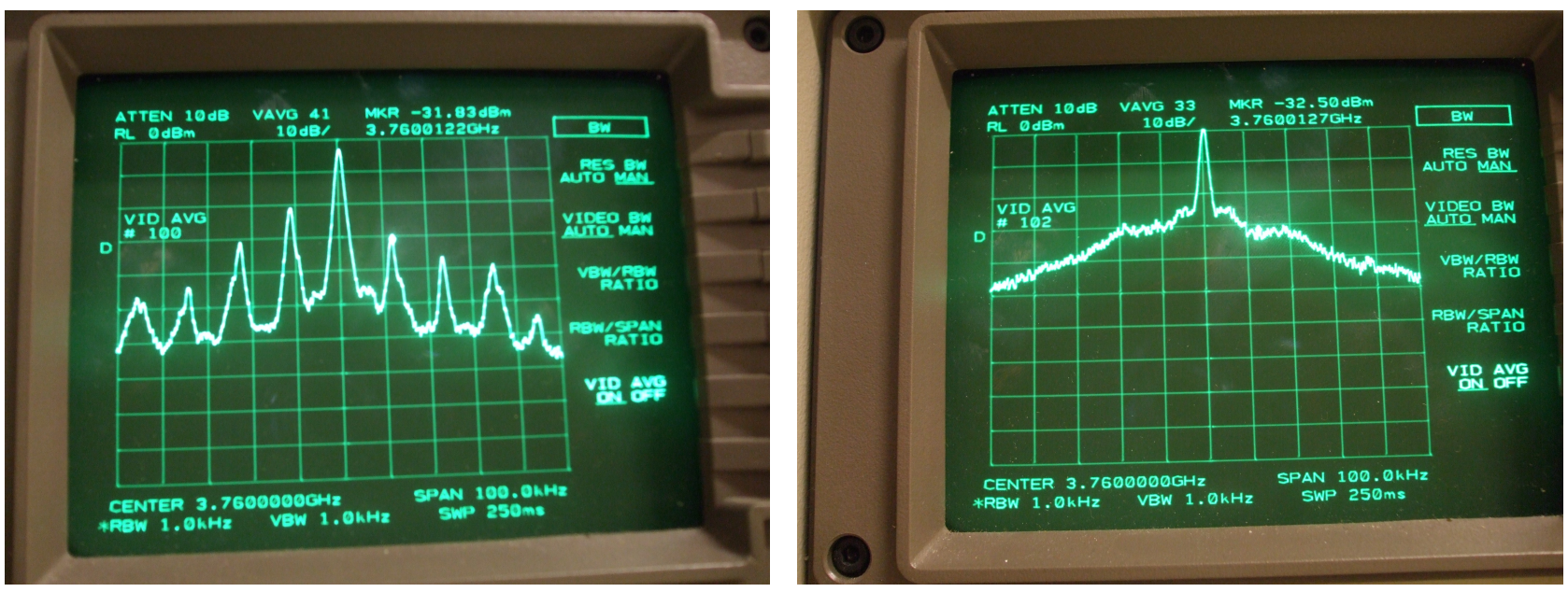

FIGURE 4. EM spectrum revealed in various regions of the plasma reactor chamber: the EM spectrum revealed near UHR (left); the EM spectrum in regions far from UHR position (right).

$\omega_{\mathrm{UH}}$ around 17, 20 and $24 \mathrm{~cm} \mathrm{LP}$ penetration length. This means that UHR exists in this position, and then EBW generation is possible.

The EM wave spectrum was studied in various positions in the plasma chamber, in particular in regions near the UHR resonance and far from it. The results, shown in Figure 4 show the strong presence of sidebands in the proximity of the calculated UHR positions, while no sidebands are revealed far away from UHR. The separation between two adjacent bands is around $10 \mathrm{kHz}$.

\section{Conclusions}

The measurements presented in this paper demonstrate that there is great scope for investigations of alternative heating schemes in an ECR-based ion source. The density can be pumped well above the cutoff value, which means that significant boosting of the output ion beam currents can be achieved in the near future. The benefits for accelerators could be valuable, considering that new ion sources may be designed with simplified magnetic field structures, requiring low levels of RF power to produce multicharged light ions (particularly important for increasing the reliability of the source).

\section{ACKNOWLEDGEMENTS}

Support for the INFN RDH-UTOPIA project and for the 5th National Committee is gratefully acknowledged. The cooperation of L. Allegra was essential for the work presented here. We acknowledge financial support from the European Union under Grant Agreement 262010ENSAR/FP7, JRA01-ARES.

\section{REFERENCES}

[1] R. Geller, Electron Cyclotron Resonance Ion source and ECR plasmas: Inst. Phys., Philadelphia, 1996.

[2] B. Wolf. Handbook of Ion Sources. FL: CRC Press, Boca Raton, 1995.
[3] S. Gammino and G. Ciavola. The role of microwave frequency on the high charge states build-up in the ECR ion sources. Plasma Source Sci. Technol., May 1996 5, 19 DOI:10.1088/0963-0252/5/1/002

[4] F. F. Chen. Introduction to the Plasma Physics and Controlled Fusion: Plasma Physics. U.K.: London Press, London, 1986.

[5] I. B. Bernstein, Phys. Rev. 109, (1958) DOI:10.1103/PhysRev.109.10

[6] K. S. Golovanivsky, V. D. Dougar-Jabon, and D. V. Reznikov, Phys. Rev. E 52, (1995) 2969. DOI:10.1103/physreve.52.2969

[7] P. Fischer, C. Gauthereau, J. Godiot, G. Matthieussent, Journal de Physique 48 (1987) 233. DOI:10.1051/jphys:01987004802023300

[8] R. Z. Sagdeev and B. D. Shapiro, Pis'ma Zh. Eksp. Teor. Fiz. 17, 389(1973).

[9] D. Mascali et al 2013 Plasma Sources Sci. Technol. 22 065006 DOI:10.1088/0963-0252/22/6/065006

[10] Y.Y. Podoba et al., Physical Review Letters 98 (2007) 255003 DOI:10.1103/PhysRevLett.98.255003

[11] J. Preinhaelter, et al., Plasma Physics and Controlled Fusion 51 (2009) 125008. DOI:10.1088/0741-3335/51/12/125008

[12] T. Taylor and J. S. C. Wills, Nucl. Instrum. Methods Phys. Res. A 309, 37 (1991). DOI:10.1016/0168-9002(91)90090-D

[13] R. Miracoli, L. Celona, G. Castro, D. Mascali, S. Gammino, Rev.Sci.Intrum. 83, (2012) 02A305 DOI:10.1063/1.3660256

[14] D. Mascali et al. Rev. Sci. Instrum. 85 , 02A956 (2014) DOI:10.1063/1.4858115.

[15] A. Gumberidze et al. Rev. Sci. Instr. 81, 033303 (2010) DOI:10.1063/1.3316805

[16] E. Canobbio. Nucl. Fusion, 1969. vol. 9, p. 27. DOI:10.1088/0029-5515/9/1/004

[17] G. Castro et al., Rev. Sci. Instr. 83, (2012) 02B501 DOI:10.1063/1.3662477.

[18] D. Mascali et al., Nucl. Instr. \& Meth. A 653 (1) 11, (2011). DOI:10.1016/j.nima.2011.02.048 